\title{
Comparison of General Practice and Pharmaceutical Dispensing Data for Pharmacoepidemiological Research: Assessing the Risk of Urinary-Tract Infections with Sodium Glucose Cotransporter 2 Inhibitors for Diabetes
}

\author{
Svetla Gadzhanova (i) and Elizabeth Roughead \\ Quality Use of Medicines and Pharmacy Research Centre, University of South Australia, Australia \\ Correspondence should be addressed to Svetla Gadzhanova; svetla.gadzhanova@unisa.edu.au
}

Received 6 September 2018; Revised 29 October 2018; Accepted 8 November 2018; Published 2 December 2018

Academic Editor: Jennifer L. Freeman

Copyright ( 2018 Svetla Gadzhanova and Elizabeth Roughead. This is an open access article distributed under the Creative Commons Attribution License, which permits unrestricted use, distribution, and reproduction in any medium, provided the original work is properly cited.

\begin{abstract}
Background. This study compared results of a study undertaken using Australia's general practice electronic health record database, MedicineInsight, to assess risk of urinary-tract infections with sodium glucose cotransporter 2 inhibitors (SGLT2) for diabetes, by undertaking the same study in a 10\% random sample of data from the national Pharmaceutical Benefits Scheme data. Methods. Cohort studies were undertaken using deidentified data from the two national datasets. In each dataset, initiators of SGLT2 inhibitors were compared to initiators of dipeptidyl peptidase 4 (DPP-4) inhibitors in the period Jan 2012 to Sep 2015. The risk of urinary-tract infections (UTI) was assessed in six-month follow-up after initiation of SGLT2 and DPP-4. Results. There were 1,977 people in the SGLT2 and 1,964 people in the DPP-4 cohort (MedicineInsight data) and 3,120 in the SGLT2 and 12,359 in the DPP-4 cohort (10\% PBS data). In both datasets, the risk of UTI after initiation of SGLT2 was not significantly increased in comparison to DPP-4 cohort (MedicineInsight: 3.6\% versus 4.9\%; aHR=0.90, 95\% CI 0.66-1.24; PBS: $3.0 \%$ versus 3.9\%; aHR=0.90, $95 \%$ CI $0.72-1.13,10 \%)$. Conclusions. Comparison of MedicineInsight data to PBS national pharmacy data demonstrated highly comparable results for the specific study question. MedicineInsight is a reliable source of data that can be used for pharmacoepidemiological studies.
\end{abstract}

\section{Introduction}

In 2011, the Australian Government Department of Health funded NPS MedicineWise "to establish and manage a longitudinal general practice data platform to improve the post-marketing surveillance of medicine use in Australia and support quality improvement activities in general practices" [1].

NPS MedicineWise began to introduce a database (MedicineInsight) from 2013 onwards, with the aim of establishing a database comprising electronic health records for postmarketing surveillance of medicines and devices. Longitudinal deidentified patient data are regularly extracted from the medical record software of 500 general practices across Australia. Over 3000 general practitioners (which is $10 \%$ of all GPs in Australia [2]) participate in the program, with data representing more than 4.8 million patients [3, 4] which is $20 \%$ of the total population in Australia in 2015 [5]. MedicineInsight data provides information on prescribed medicines, pathology results and clinical observation, and diagnosis/reason for the GP visit. For all prescribed medicine, the prescribing date, type, dose, strength, quantity, and number of repeats are recorded as well as reason for prescribing. This has the potential to be a significant advance of existing Australian datasets for pharmacoepidemiological studies and health service research as the data include clinical information.

The existing datasets in Australia were administrative health claims data, namely, the Medicare Benefits Schedule data, which is a national claims dataset for medical practitioner attendances (with no reason for encounter) and diagnostic and pathology services provided (with no clinical 
data), and the Pharmaceutical Benefits Scheme (PBS) data, which includes records of all subsidised medicines under the national scheme for treatment of most health conditions of all Australian residents. PBS data are collected from community pharmacies, private hospitals, and most public hospitals. Patient information includes gender, age, beneficiary status (general or concessional), and geographic location. Prescription information include date of supply, drug code, therapeutic class, generic name, form, quantity dispensed, and number of prescription repeats. Since the middle of 2012, PBS data represents full capture of dispensing records for both general and concessional beneficiaries. One advantage of the PBS dataset over the MedicineInsight dataset is the frequency of records for prescription medicines. In Australia, the majority of medicines for chronic conditions are supplied in quantities of one month's supply with pharmacists allowed to refill the prescription another five times, providing six month's supply in total with a record in the dataset approximately every month. By comparison MedicineInsight data has the record of the original prescription; thus, unless changes to medicines are made, one record appears approximately every six months. Another difference between the two datasets is that the Pharmaceutical Benefits Scheme data include all 24 million persons in Australia, while MedicineInsight data involve a sample of patients based on GP practices volunteering to participate.

Given that MedicineInsight data contains health records, it has the potential to be used for health outcome studies and other advanced pharmacoepidemiological research in Australia. The data for the database is sourced from voluntary participation of general practices. While the number of practices participating is large, resulting in a large number of patients, whether the data gives consistent results with other Australian datasets is unknown. For this reason, we chose to triangulate the results from a study using the MedicineInsight database with the results using the same study design but using the national PBS dataset. We chose to use the national PBS dataset for comparison as it represents complete capture of medicines information for all Australians (24 million persons), as all Australians are provided with medicines via the national Pharmaceutical Benefits Scheme. It would be advantageous to determine the comparative performance of the MedicineInsight and PBS datasets because they are complementary with regard to their data collection on medicine utilisation, yet their source data are substantially different. Thus, consistent findings from both datasets gives more support to observational research findings. In this research, we aimed to compare the results of a study undertaken using Australia's general practice electronic health record database, MedicineInsight, to assess risk of urinary-tract infections with sodium glucose cotransporter 2 inhibitors for diabetes [6] by undertaking the same study in a $10 \%$ random sample of data from the national Pharmaceutical Benefits Scheme data. This example was chosen, as one of the main purposes of establishing the MedicineInsight data collection was to improve the postmarketing surveillance of medicines in Australia. The SGLT 2 inhibitors were subject to a risk management plan to monitor safety, including the incidence of urinary infections, when they were approved for marketing in Australia by the Australian Therapeutic Goods Administration.

\section{Materials and Methods}

2.1. Study Populations. Retrospective longitudinal studies were conducted utilising the two national datasets. In both datasets all patients with a SGLT2 inhibitor initiation between 1 Jan 2013 and 1 Sep 2015 were identified. A new user design was used which included all patients with prior history in the 12 months prior to the first prescription of an SGLT2 or DPP4. Initiation was defined as no record of any SGLT2 or DPP4 medicine in the previous 12 months. For MedicineInsight data prior history was defined as having at least one visit to the GP in the 12 months prior to medicine initiation, while for the PBS data it was defined as having at least one dispensing for any medicine. All people who initiated dipeptidyl peptidase 4 (DPP-4) inhibitor in the same period were included as a comparison cohort. The date of initiation of either SGLT2 or DPP-4 inhibitors was considered as index date. People who were initiated on both products within 18 months were excluded to avoid overlap of pre- and post-periods.

2.2. Medicines Included in the Analysis. There are three SGLT2 inhibitors available in Australia. The Anatomical Therapeutic Chemical (ATC) code [2] was used to identify them. Dapagliflozin is available as $10 \mathrm{mg}$ only (ATC code: A10BX09); Empagliflozin is available as $10 \mathrm{mg}$ and $25 \mathrm{mg}$ (ATC code A10BX12); and Canagliflozin is available as $100 \mathrm{mg}$ or $300 \mathrm{mg}$ (ATC code A10BX11).

Saxagliptin, sitagliptin, linagliptin vildagliptin, alogliptin, and linagliptin (either as a single agent product or a combination product) are the dipeptidyl peptidase 4 inhibitors available in Australia. They were selected by ATC code A10BH and A10BD07- A10BD11.

2.3. Statistical Analysis. We utilised both MedicineInsight and the $10 \%$ PBS dataset to investigate the strength of the SGLT2 inhibitor at initiation and the use of other diabetes medicines in the year prior to index SGLT2 as well as the use of potentially interacting medicines. Angiotensin Converting Enzyme (ACE) inhibitors, Angiotensin II receptor blockers (ARB), nonsteroidal anti-inflammatory drugs (NSAIDs), and loop diuretics may potentially interact with the SGLT2 inhibitors and their use was analysed [6]. Concurrent use of interacting medicines was defined as a record of a prescription in the three months prior to or the six-month period after initiation of the SGLT2 or DPP 4 inhibitors. This period of time was chosen as PBS prescriptions are for one-month supply and allow for up to five repeats; thus a six-month period is the minimum period required to see a record in the prescribing dataset.

The risk of urinary-tract infections (UTIs) was the main outcome measure of interest. It was assessed for the 6 months after SGLT2 and DPP-4 initiation. For MedicineInsight data we investigated prescribing of UTI specific medicines (trimethoprim, nitrofurantoin, norfloxacin) as well as reasons for the visit to the GP and recorded diagnosis/condition 
TABLE 1: Cohorts' demographics.

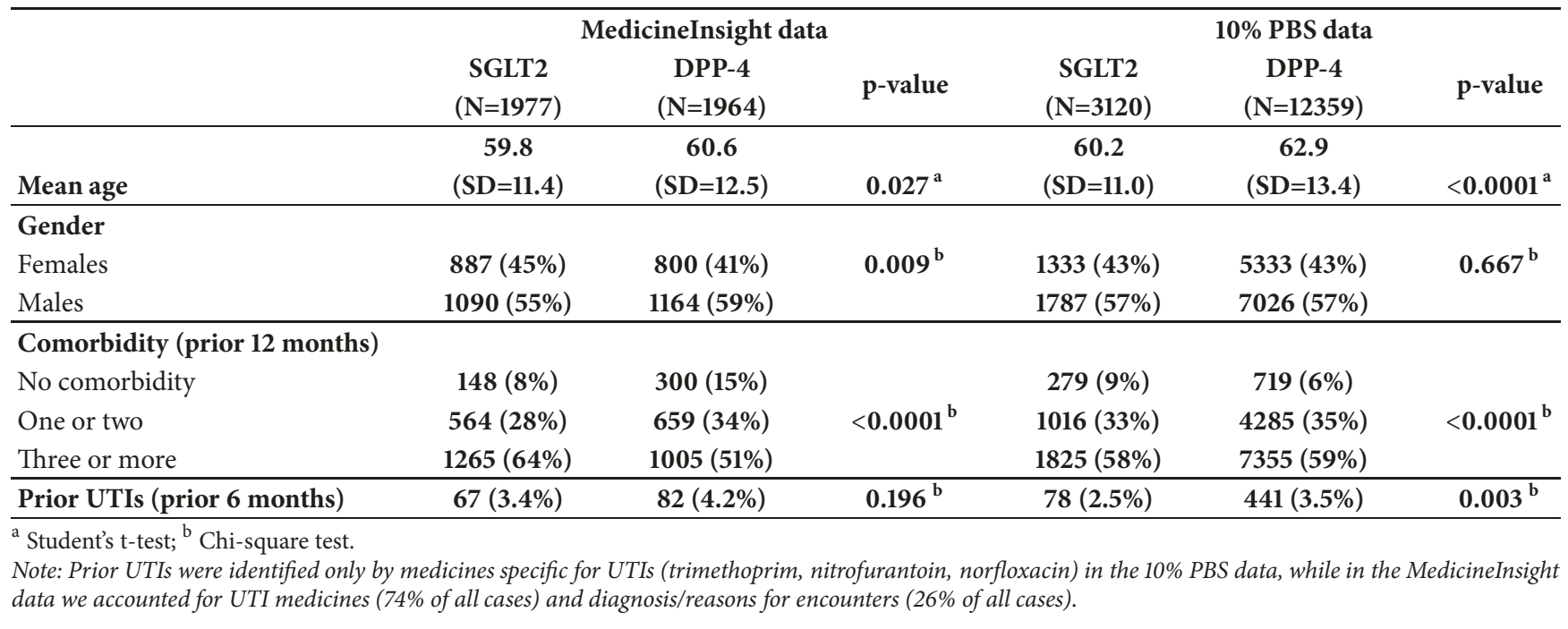

using definite terms to identify urinary-tract infections. For PBS data we investigated only dispensing of UTI specific medicines (trimethoprim, nitrofurantoin, norfloxacin) as no diagnostic or encounter information was available. Furthermore, we performed sensitivity analysis and assessed the risk of UTIs in subjects who were only prescribed the three medicines specific for UTI in MedicineInsight data (i.e., excluding UTIs identified from clinical information).

Demographic characteristics of the cohorts from the two datasets were compared, including mean age, gender, number of comorbidities, and percent of prior infections. Comorbidities were determined based on prescription history using the Rx-Risk-V Comorbidity index [7]. Proportions were compared by chi-square tests and means were compared by Student's t-test.

Median time from initiation of SGLT2 or DPP-4 to first UTI in the follow-up period was determined using Kaplan Meier method. People were censored for death and end of study in the MedicineInsight data and only for end of study in the $10 \%$ PBS data as date of death is unavailable in the PBS dataset. Cox proportional hazards models were used to determine hazard ratios. The models were adjusted for gender, age, comorbidity, and prior UTI infections. All data analyses were performed using SAS software (version V9.4, SAS Institute Inc. Cary, North Caroline, USA).

The study was approved by the NPS MedicineInsight Data Governance Committee and the Australian Government Department of Human Services External Requests Evaluation Committee.

\section{Results}

There were 1,977 people initiated on SGLT2 inhibitors in the MedicineInsight data and 3,120 in the PBS data. There were 1,964 patients initiated on DPP-4 in MedicineInsight data and 12,359 in the PBS data. Demographics of the cohorts are presented in Table 1 . In both datasets, the SGLT2 cohort was younger and had more comorbidities than the DPP4 cohort. The rates of prior UTI were slightly higher in MedicineInsight data but that could be due to the availability of diagnostic/encounter information in addition to prescribing information which accounted for one-quarter of the identified prior UTIs.

In both datasets, dapagliflozin accounted for the majority of SGLT2 initiation (Table 2). However, canagliflozin was initiated in higher proportion of people in PBS data $(9 \%$ versus $3 \%$ in MedicineInsight, chi-square $\mathrm{p}<0.0001)$. The SGLT2 inhibitors strength use was similar in the two datasets. Vast proportion of SGLT2 initiators had prior diabetes therapy (90\% in MedicineInsight and $80 \%$ in PBS), and similar proportions were coprescribed potentially interacting medicines (Table 2).

Post-UTIs were identified only by medicines specific for UTIs (trimethoprim, nitrofurantoin, norfloxacin) in the $10 \%$ PBS data, while in the MedicineInsight data we accounted for UTI medicines (74\% of all cases) and diagnosis/reasons for encounters (26\% of all cases). The frequency of urinary-tract infections after SGLT2 initiation was low: majority of people who had a UTI had just one occurrence in the 6 months after SGLT2 initiation happening in the first 12 weeks (Table 3 ).

In both datasets, the risk of UTIs was not increased in the SGLT2 initiators compared to DPP-4 initiators (Table 4):

(i) for MedicineInsight data: 3.6\% versus $4.9 \%$, adjusted hazard ratio $(\mathrm{aHR})=0.90,95 \%$ CI $0.66-1.24$;

(ii) for $10 \%$ PBS data: $3.0 \%$ versus $3.9 \%$, aHR $=0.90,95 \%$ CI 0.72-1.13.

Sensitivity analysis for MedicineInsight data assessing the risk of UTIs based only on the medicines specific for UTIs resulted in $\mathrm{aHR}=1.02,95 \% \mathrm{CI}$ 0.77-1.65.

\section{Discussion}

This study is the first to our knowledge to compare MedicineInsight results against another national dataset. In 
TABLE 2: Other indicators.

\begin{tabular}{|c|c|c|}
\hline & $\begin{array}{c}\text { MedicineInsight } \\
\text { SGLT2 cohort }(\mathrm{N}=1977)\end{array}$ & $\begin{array}{c}10 \% \text { PBS } \\
\text { SGLT2 cohort }(\mathrm{N}=3120)\end{array}$ \\
\hline \multirow{3}{*}{ Type of the index medicine } & Dapagliflozin - 93\% & Dapagliflozin - 87\% \\
\hline & Canagliflozin - 3\% & Canagliflozin - 9\% \\
\hline & Empagliflozin - 4\% & Empagliflozin $-4 \%$ \\
\hline \multirow{3}{*}{ Strength at initiation } & $\begin{array}{c}\text { Dapagliflozin initiators - all on 10mg } \\
\text { (one strength) }\end{array}$ & $\begin{array}{l}\text { Dapagliflozin initiators - all on 10mg } \\
\text { (one strength) }\end{array}$ \\
\hline & $\begin{array}{l}\text { Canagliflozin initiators }-78 \% \text { on } \\
\text { 300mg (higher strength) }\end{array}$ & $\begin{array}{c}\text { Canagliflozin initiators }-77 \% \text { on } \\
\text { 300mg (higher strength) }\end{array}$ \\
\hline & $\begin{array}{l}\text { Empagliflozin initiators }-74 \% \text { on } 10 \mathrm{mg} \\
\text { (lower strength) }\end{array}$ & $\begin{array}{c}\text { Empagliflozin initiators }-72 \% \text { on } 10 \mathrm{mg} \\
\text { (lower strength) }\end{array}$ \\
\hline \multirow{2}{*}{$\begin{array}{l}\text { Prior diabetes therapy } \\
\text { (prior } 12 \text { months) }\end{array}$} & $\begin{array}{l}\text { 90\% had documented diabetes } \\
\text { medicine }\end{array}$ & $\begin{array}{l}80 \% \text { had dispensing for diabetes } \\
\text { medicine }\end{array}$ \\
\hline & $\begin{array}{l}86 \% \text { had metformin and/or } \\
\text { sulfonylurea }\end{array}$ & $\begin{array}{l}69 \% \text { had metformin and/or } \\
\text { sulfonylurea }\end{array}$ \\
\hline \multirow{3}{*}{$\begin{array}{l}\text { Interacting medicines } \\
(3 \text { months prior and } 6 \text { months post } \\
\text { index) }\end{array}$} & ACE/ARB - 61\% & ACE/ARB - 61\% \\
\hline & NSAIDs - $14 \%$ & NSAIDs - $14 \%$ \\
\hline & Frusemide $-6 \%$ & Frusemide - 7\% \\
\hline
\end{tabular}

TABLE 3: Frequency of UTIs in the 6 months after SGLT2 or DPP-4 initiation.

\begin{tabular}{|c|c|c|c|c|}
\hline & \multicolumn{2}{|c|}{ MedicineInsight data } & \multicolumn{2}{|c|}{$10 \%$ PBS data } \\
\hline & SGLT2 & DPP-4 & SGLT2 & DPP-4 \\
\hline & $(\mathrm{N}=1977)$ & $(\mathrm{N}=1964)$ & $(\mathrm{N}=3120)$ & $(\mathrm{N}=12359)$ \\
\hline \multirow{4}{*}{$\begin{array}{l}\text { Proportion with at } \\
\text { least one UTI }\end{array}$} & $3.6 \%(\mathrm{~N}=71)$ & $4.9 \%(\mathrm{~N}=97)$ & $3.0 \%(\mathrm{~N}=94)$ & $3.9 \%(\mathrm{~N}=479)$ \\
\hline & Of those: & Of those: & Of those: & Of those: \\
\hline & $\begin{array}{l}89 \% \text { had just one } \\
\text { infection; }\end{array}$ & $\begin{array}{l}91 \% \text { had just one } \\
\text { infection; }\end{array}$ & $\begin{array}{l}83 \% \text { had just one } \\
\text { infection; }\end{array}$ & $\begin{array}{l}80 \% \text { had just one } \\
\text { infection; }\end{array}$ \\
\hline & $\begin{array}{c}64 \% \text { had the } 1^{\text {st }} \text { infection } \\
\text { in the } 12 \text { weeks post } \\
\text { index SGLT2 }\end{array}$ & $\begin{array}{c}58 \% \text { had the } 1^{\text {st }} \\
\text { infection in the } 12 \\
\text { weeks post index DPP-4 }\end{array}$ & $\begin{array}{c}64 \% \text { had the } 1^{\text {st }} \text { infection } \\
\text { in the } 12 \text { weeks post } \\
\text { index SGLT2 }\end{array}$ & $\begin{array}{c}59 \% \text { had the } 1^{\text {st }} \\
\text { infection in the } 12 \\
\text { weeks post index DPP-4 }\end{array}$ \\
\hline
\end{tabular}

this study we compared results from MedicineInsight data on SGLT2 inhibitors for type 2 diabetes and risk of infections [6] with results from the $10 \%$ PBS data. We investigated the use of prior diabetes therapies and interacting medicines, as well as safety concerns related to risk of urinary-tract infections. We found very similar results across both datasets, with a very high proportion of SGLT2 initiators having prior diabetes therapy, which is consistent with subsidy requirements, almost identical proportions receiving the potentially interacting medicines, and very similar but not significant hazard ratios for the risk of UTI post SGLT2 initiation. MedicineInsight data is superior to PBS data as it provides not only information on prescribed medicines but also clinical information about reasons for encounters and diagnosis (e.g., diagnoses for UTI), as well as pathology/laboratory results (e.g., HbAlc test results) and observation data (e.g., weight). This may account for some differences in the rates of prior and post-UTIs (slightly higher in MedicineInsight than in the PBS data) as diagnosis/reasons for encounters contributed to identification of extra patients with a UTI. However, the hazard ratios for the risk of UTI after SGLT2 initiation were very similar and nonsignificant in both datasets. Sensitivity analysis for MedicineInsight data assessing the risk of UTIs based only on the three medicines specific for UTIs resulted in a similar and nonsignificant hazard ratio.

Our results are consistent with existing evidence on UTI risk associated with use of SGLT2 inhibitors. With regard to dapagliflozin, the rate of UTI was not significantly increased in 12 randomized placebo-controlled trials ( $4.3 \%$ versus $3.7 \%$, $\mathrm{p}=0.48$ ) [8]. Meta-analyses of the use of SGLT2 inhibitors in comparison with insulin for diabetes found no increased risk of urinary-tract infections [9]. An indirect comparison between SGLT2 inhibitors (added to insulin) and DPP4 inhibitors (added to insulin) from fourteen randomized controlled trials showed no differences in the risk of UTIs between SGLT2/insulin and DPP-4/insulin ( $R R=1.38,95 \% \mathrm{CI}$ $0.87-2.19, \mathrm{p}=0.149)$ [10]. 
TABLE 4: Risk of UTIs.

\begin{tabular}{lcccc}
\hline & \multicolumn{2}{c}{ MedicineInsight data } & \multicolumn{2}{c}{ 10\% PBS data } \\
\hline & $\begin{array}{c}\text { Unadjusted Hazard } \\
\text { ratio (HR), }\end{array}$ & $\begin{array}{c}\text { Adjusted Hazard ratio } \\
\text { (aHR), }\end{array}$ & $\begin{array}{c}\text { Unadjusted Hazard } \\
\text { ratio (HR), }\end{array}$ & $\begin{array}{c}\text { Adjusted Hazard ratio } \\
(\text { aHR), }\end{array}$ \\
& $95 \%$ CI & $95 \%$ CI & $95 \%$ CI & $95 \%$ CI \\
\hline SGLT2 vs DPP-4 cohort & $0.88(0.65-1.19)$ & $0.90(0.66-1.24)$ & $0.75(0.60-0.94)$ & $0.90(0.72-1.13)$ \\
\hline
\end{tabular}

Note: In the 10\% PBS data the adjusted hazard ratio (aHR) is adjusted for prior infection, age, gender, and comorbidity; in the MedicineInsight data the adjusted hazard ratio (aHR) is adjusted for prior infection, age, gender, weight, HbAlc, location, smoking status, and comorbidity.

A strength of this study is the direct comparison of two large national datasets on a number of indicators: one representing 500 general practices, 3000 GPs, and 4.8 million patients; the other capturing prescription data of $10 \%$ (2.5 million persons) of all Australians. Limitation of both datasets is the lack of information if the prescribed medicines were actually consumed. MedicineInsight provides no linkage information for patients visiting multiple GP practices which might lead to loss of patients to follow-up. This may indicate potential underestimation of the outcome events. While MedicineInsight data provide clinical and diagnosing information allowing identification of all patients with a UTI, the PBS data do not include diagnostic or encounter information which can result in underestimation of the outcome events in the PBS population. Another limitation is that the analysis based on PBS data was not censoring for death as these data were unavailable in the PBS dataset.

Even though the risk of genital infections has been assessed in MedicineInsight data, it was not possible to be assessed in the PBS data as fungal creams and tablets are not generally subsidised on the PBS and there is no diagnostic or encounter information in the data. This confirms again that MedicineInsight data is a more complete source of medical information.

\section{Conclusion}

Comparison of MedicineInsight data to PBS national pharmaceutical data demonstrated highly comparable results for the specific study question. MedicineInsight is a rich dataset providing clinical and diagnostic information as well as observation and pathology data in addition to pharmaceutical claims which makes it a valuable data source for pharmacoepidemiological research.
Abbreviations
ACE: Angiotensin Converting Enzyme
ARB: Angiotensin II receptor blockers
ATC: Anatomical Therapeutic Chemical
DPP-4: Dipeptidyl peptidase 4
GP: $\quad$ General practitioners
NSAID: Nonsteroidal anti-inflammatory drugs
PBS: Pharmaceutical Benefits Scheme
SGLT2: Sodium glucose cotransporter 2
UTI: Urinary-tract infections.

\section{Data Availability}

The provision of information to third parties with an interest in MedicineInsight data is subject to a rigorous and formal approval process and is guided by the MedicineInsight independent external Data Governance Committee. This Committee includes GPs, consumer advocates, privacy experts, and researchers.

\section{Ethical Approval}

The PBS study used deidentified data and conforms to management and release of data in accordance with the principles of the Australian Government Privacy Act, 1988.

\section{Disclosure}

The authors declare that NPS had no involvement in the study design, in the analysis and data interpretation, in the manuscript writing, and in the decision to submit for publication.

\section{Conflicts of Interest}

The authors declare no conflicts of interest.

\section{Acknowledgments}

The study was part of the employment of the authors by the University of South Australia. The data for the study was provided by NPS MedicineWise.

\section{References}

[1] NPS MedicineWise, "Using MedicineInsight data," 2017. https:// www.nps.org.au/medicine-insight/using-medicineinsight-data.

[2] "Drug utilisation sub-committee (DUSC)," Medicines for the treatment of diabetes, 2017, http://www.pbs.gov.au/info/ industry/listing/participants/public-release-docs/2017-02/medicines-diabetes-feb-2017.

[3] "MedicineInsight: Representativeness and overview of MedicineInsight data," NPS MedicineWise, Sydney, 2016.

[4] NPS MedicineWise, "What is MedicineInsight?" 2015. http:// www.nps.org.au/health-professionals/medicineinsight.

[5] "Australian Bureau of Statistics," Australian Demographic Statistics, 2015, http://www.abs.gov.au/ausstats/abs@.nsf/mf/3101.0. 
[6] S. Gadzhanova, N. Pratt, and E. Roughead, "Use of SGLT2 inhibitors for diabetes and risk of infection: Analysis using general practice records from the NPS MedicineWise MedicineInsight program," Diabetes Research and Clinical Practice, vol. 130, pp. 180-185, 2017.

[7] A. Vitry, S. A. Wong, E. E. Roughead, E. Ramsay, and J. Barratt, "Validity of medication-based co-morbidity indices in the Australian elderly population," Australian and New Zealand Journal of Public Health, vol. 33, no. 2, pp. 126-130, 2009.

[8] K. M. Johnsson, A. Ptaszynska, B. Schmitz, J. Sugg, S. J. Parikh, and J. F. List, "Urinary tract infections in patients with diabetes treated with dapagliflozin," Journal of Diabetes and its Complications, vol. 27, no. 5, pp. 473-478, 2013.

[9] Y. Yang, S. Chen, H. Pan et al., "Safety and efficiency of SGLT2 inhibitor combining with insulin in subjects with diabetes," Medicine, vol. 96, no. 21, p. e6944, 2017.

[10] S. H. Min, J.-H. Yoon, S. Hahn, and Y. M. Cho, "Comparison between SGLT2 inhibitors and DPP4 inhibitors added to insulin therapy in type 2 diabetes: a systematic review with indirect comparison meta-analysis," Diabetes/Metabolism Research and Reviews, vol. 33, no. 1, 2017. 


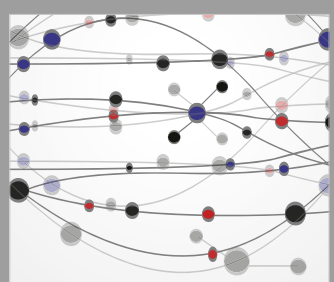

The Scientific World Journal
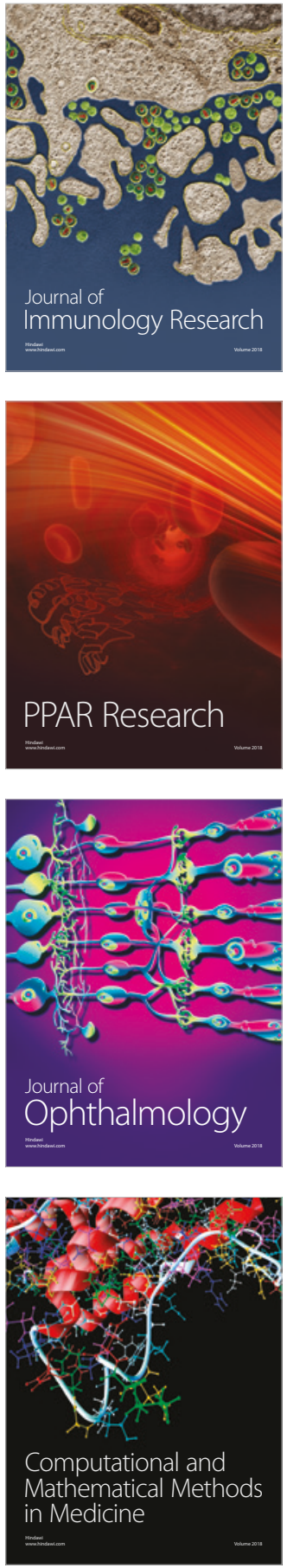

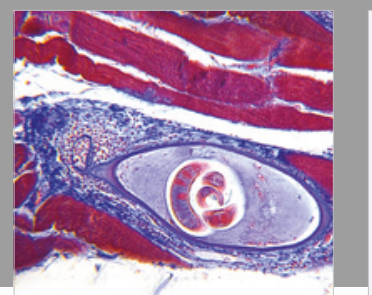

Gastroenterology Research and Practice

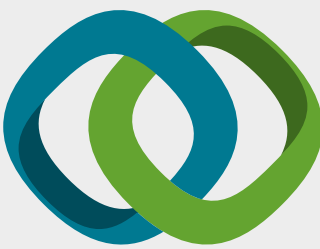

\section{Hindawi}

Submit your manuscripts at

www.hindawi.com
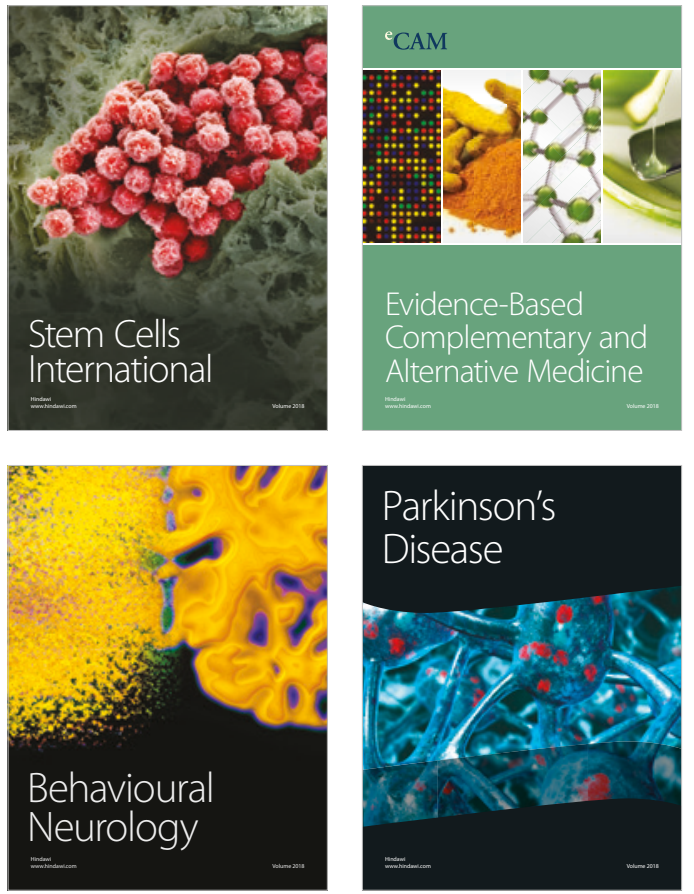

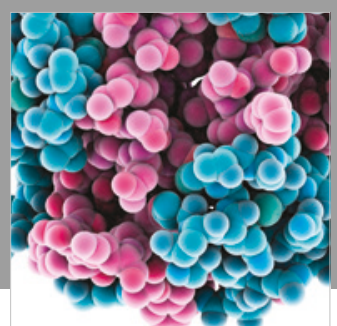

ournal of

Diabetes Research

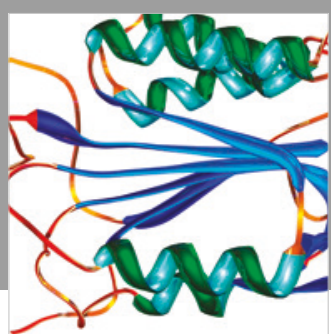

Disease Markers
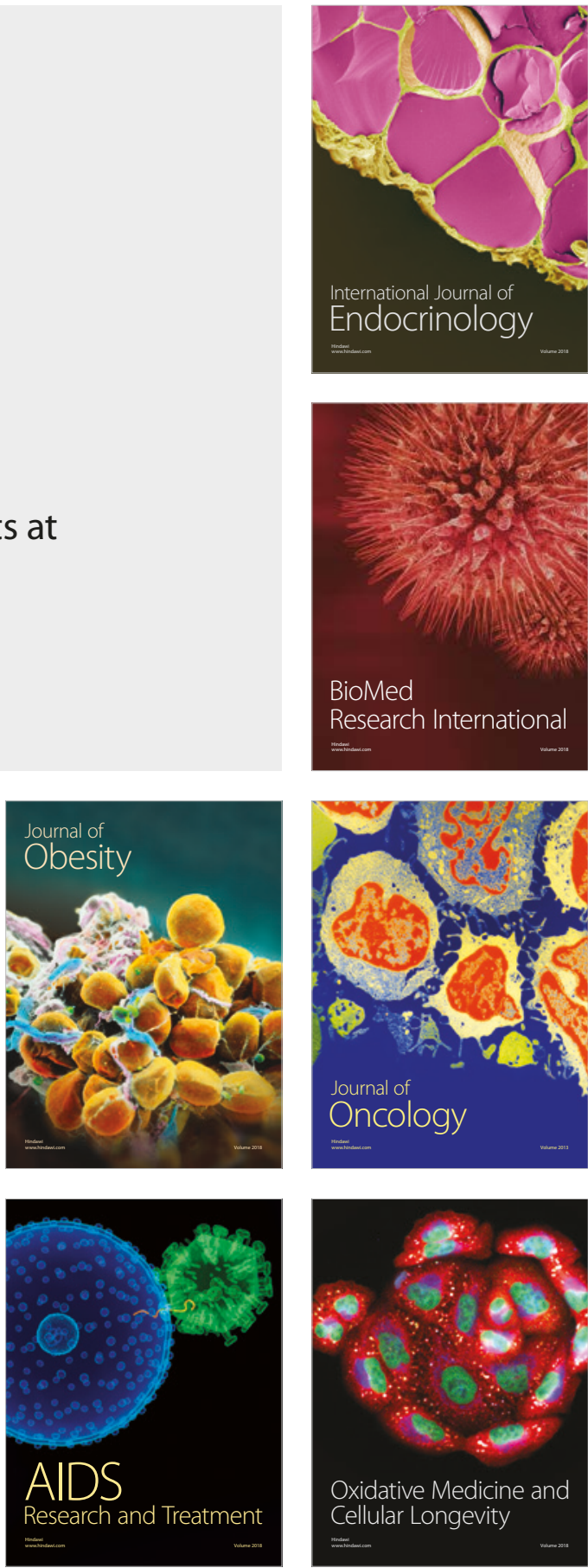